$\mathrm{J}$ o u r n a l of

Mathematics

and Applications

JMA No 40, pp 69-84 (2017)

\title{
Measures of Noncompactness in a Banach Algebra and Their Applications
}

\author{
Szymon Dudek
}

\begin{abstract}
In this paper we study the existence of solutions of a nonlinear quadratic integral equation of fractional order. This equation is considered in the Banach space of real functions defined, continuous and bounded on the real half axis. Additionally, using the technique of measures of noncompactness we obtain some characterization of considered integral equation. We provide also an example illustrating the applicability of our approach.
\end{abstract}

AMS Subject Classification: 45G10, 47H08, 47H10.

Keywords and Phrases: Banach algebra; Measure of noncompactness; Integral equation of fractional order; Fixed point theorem; Asymptotic stability; Ultimate monotonicity.

\section{Introduction}

Nonlinear functional-integral equations are often applicable in medicine, engineering, mathematical physics, radiative transfer, kinetic theory of gases and so on (cf. $[3,13$, 14, 15, 16, 17, 20, 21], for example).

The purpose of the paper is to study the solvability of nonlinear quadratic integral equation of fractional order in the Banach algebra $B C\left(\mathbb{R}_{+}\right)$consisting of real, continuous and bounded functions defined on an unbounded interval. The equation considered in this paper can be written as

$$
x(t)=\left(U_{1} x\right)(t)\left(U_{2} x\right)(t),
$$

where

$$
\left(U_{i} x\right)(t)=m_{i}(t)+f_{i}(t, x(t)) \int_{0}^{t} \frac{v_{i}(t, s, x(s))}{(t-s)^{\alpha_{i}}} d s
$$

for $t \in \mathbb{R}_{+}, \alpha_{i} \in(0,1), i=1,2$. Moreover, $m_{i}, f_{i}, v_{i}$ are functions satisfying certain conditions for $i=1,2$.

COPYRIGHT (c) by Publishing House of Rzeszów University of Technology P.O. Box 85, 35-959 Rzeszów, Poland 
Notice that differential and integral equations of fractional order create an important and significant branch of nonlinear analysis and the theory of integral equations (cf. $[7,8,10,16])$.

Functional integral equations considered in Banach algebras have rather complicated form and the study of such equations requires of the use of sophisticated tools (cf. $[6,7,11,12,18,19]$ ). We will use the technique associated with measures of noncompactness and some fixed point theorems [5]. The so-called condition $(m)$ (introduced in [7]) related to the operation of multiplication in the algebra will be crucial in our considerations.

Measure of noncompactness used here allows us not only to obtain the existence of solutions of functional integral equation but also to characterize those solutions in terms of asymptotic stability and ultimate monotonicity.

The paper is a corrected and improved version of [4].

\section{Notation, definitions and auxiliary results}

In this section we collect some basic definitions and facts which will be used further on. At the beginning we introduce some notation.

Denote by $\mathbb{R}$ the set of real numbers and put $\mathbb{R}_{+}=[0, \infty)$. Let $(E,\|\cdot\|)$ be a Banach space with zero element $\theta$. Then by $B(x, r)$ we denote the closed ball centered at $x$ and with radius $r$. The symbol $B_{r}$ stands for the ball $B(\theta, r)$. If $X$ is a subset of $E$, we use $\bar{X}$ and $\operatorname{Conv} X$ to denote the closure and convex closure of $X$, respectively. Apart from this the symbol $\operatorname{diam} X$ will denote the diameter of a bounded set $X$ while $\|X\|$ denotes the norm of $X$ i.e., $\|X\|=\sup \{\|x\|: x \in X\}$.

Next, let us denote by $\mathfrak{M}_{E}$ the family of all nonempty and bounded subsets of $E$ and by $\mathfrak{N}_{E}$ its subfamily consisting all relatively compact sets. We use the following definition of the measure of noncompactness given in [5].

Definition 2.1. A mapping $\mu: \mathfrak{M}_{E} \rightarrow \mathbb{R}_{+}$will be called a measure of noncompact$n e s s$ in $E$ if it satisfies the following conditions:

$1^{\circ}$ The family $\operatorname{ker} \mu=\left\{X \in \mathfrak{M}_{E}: \mu(X)=0\right\}$ is nonempty and ker $\mu \subset \mathfrak{N}_{E}$.

$2^{\circ} \quad X \subset Y \Rightarrow \mu(X) \leq \mu(Y)$.

$3^{\circ} \mu(\bar{X})=\mu(\operatorname{Conv} X)=\mu(X)$.

$4^{\circ} \mu(\lambda X+(1-\lambda) Y) \leq \lambda \mu(X)+(1-\lambda) \mu(Y)$ for $\lambda \in[0,1]$.

$5^{\circ}$ If $\left(X_{n}\right)$ is a sequence of closed sets from $\mathfrak{M}_{E}$ such that $X_{n+1} \subset X_{n}$ for $n=1,2, \ldots$ and if $\lim _{n \rightarrow \infty} \mu\left(X_{n}\right)=0$, then the set $X_{\infty}=\bigcap_{n=1}^{\infty} X_{n}$ is nonempty.

The family ker $\mu$ described in $1^{\circ}$ is said to be the kernel of the measure of noncompactness $\mu$.

It is easy to show that the set $X_{\infty}$ from the axiom $5^{\circ}$ is a member of the family ker $\mu$. Indeed, from the inequality $\mu\left(X_{\infty}\right) \leq \mu\left(X_{n}\right)$ being satisfied for all $n=1,2, \ldots$ 
we derive that $\mu\left(X_{\infty}\right)=0$ which means that $X_{\infty} \in \operatorname{ker} \mu$. This fact will play a key role in our further considerations.

In the sequel we will usually assume that the space $E$ has the structure of Banach algebra. Then we write $x y$ in order to denote the product of elements $x, y \in E$. Similarly, we will write $X Y$ to denote the product of subsets $X, Y$ of $E$ i.e., $X Y=$ $\{x y: x \in X, y \in Y\}$.

Now, we recall a useful concept (see [7]).

Definition 2.2. We say that the measure of noncompactness $\mu$ defined on the Banach algebra $E$ satisfies condition $(m)$ if for arbitrary sets $X, Y \in \mathfrak{M}_{E}$ the following inequality is satisfied:

$$
\mu(X Y) \leq\|X\| \mu(Y)+\|Y\| \mu(X)
$$

It turns out that the above defined condition $(m)$ is very convenient in considerations connected with the use of the technique of measures of noncompactness in Banach algebras.

For our purposes we will need a fixed point theorem for operators acting in a Banach algebra and satisfying some conditions expressed with help of a measure of noncompactness. To this end we first recall a concept parallel to the concept of Lipschitz continuity (cf. [5]).

Definition 2.3. Let $\Omega$ be a nonempty subset of a Banach space $E$ and let $F: \Omega \rightarrow E$ be a continuous operator which transforms bounded subsets of $\Omega$ onto bounded ones. We say that $F$ satisfies the Darbo condition with a constant $k$ with respect to a measure of noncompactness $\mu$ if $\mu(F X) \leq k \mu(X)$ for each $X \in \mathfrak{M}_{E}$ such that $X \subset \Omega$. If $k<1$, then $F$ is called a contraction with respect to $\mu$.

Now, assume that $E$ is a Banach algebra and $\mu$ is a measure of noncompactness on $E$ satisfying condition $(m)$. Then we have the following theorem announced above $[7]$.

Theorem 2.4. Assume that $\Omega$ is nonempty, bounded, closed and convex subset of the Banach algebra $E$, and the operators $P$ and $T$ transform continuously the set $\Omega$ into $E$ in such a way that $P(\Omega)$ and $T(\Omega)$ are bounded. Moreover, we assume that the operator $F=P \cdot T$ transforms $\Omega$ into itself. If the operators $P$ and $T$ satisfy on the set $\Omega$ the Darbo condition with respect to the measure of noncompactness $\mu$ with the constants $k_{1}$ and $k_{2}$ respectively, then the operator $F$ satisfies on $\Omega$ the Darbo condition with the constant

$$
\|P(\Omega)\| k_{2}+\|T(\Omega)\| k_{1}
$$

Particularly, if $\|P(\Omega)\| k_{2}+\|T(\Omega)\| k_{1}<1$, then $F$ is a contraction with respect to the measure of noncompactness $\mu$ and has at least one fixed point in the set $\Omega$.

Remark 2.5. It can be shown [5] that the set Fix $F$ of all fixed points of the operator $F$ on the set $\Omega$ is a member of the kernel ker $\mu$. 


\section{Some measure of noncompactness in the Banach algebra $B C\left(\mathbb{R}_{+}\right)$}

In this section we present some measures of noncompactness in the Banach algebra $B C\left(\mathbb{R}_{+}\right)$consisting of all real functions defined, continuous and bounded on the half axis $R_{+}$. The algebra $B C\left(\mathbb{R}_{+}\right)$is endowed with the usual supremum norm

$$
\|x\|=\sup \left\{|x(t)|: t \in \mathbb{R}_{+}\right\}
$$

for $x \in B C\left(\mathbb{R}_{+}\right)$. Obviously, the multiplication in $B C\left(\mathbb{R}_{+}\right)$is understood as the usual product of real functions. Let us mention that measures of noncompactness which we intend to present here, were considered in details in [7].

In what follows let us assume that $X$ is an arbitrarily fixed nonempty and bounded subset of the Banach algebra $B C\left(\mathbb{R}_{+}\right)$i.e., $X \in \mathfrak{M}_{B C\left(\mathbb{R}_{+}\right)}$. Choose arbitrary $\varepsilon>0$ and $T>0$. For $x \in X$ denote by $\omega^{T}(x, \varepsilon)$ the modulus of continuity of the function $x$ on the interval $[0, T]$ i.e.,

$$
\omega^{T}(x, \varepsilon)=\sup \{|x(t)-x(s)|: t, s \in[0, T],|t-s| \leq \varepsilon\} .
$$

Next, let us put:

$$
\begin{gathered}
\omega^{T}(X, \varepsilon)=\sup \left\{\omega^{T}(x, \varepsilon): x \in X\right\}, \\
\omega_{0}^{T}(X)=\lim _{\varepsilon \rightarrow 0} \omega^{T}(X, \varepsilon), \\
\omega_{0}^{\infty}(X)=\lim _{T \rightarrow \infty} \omega_{0}^{T}(X) .
\end{gathered}
$$

In our considerations we will also need another set quantities. In order to define this quantities, let us fix $t \in \mathbb{R}_{+}$and denote by $X(t)$ the cross-section of the set $X$ at the point $t$ i.e., $X(t)=\{x(t): x \in X\}$. Denote by $\operatorname{diam} X(t)$ the diameter of $X(t)$. Further, for a fixed $T>0$ and $x \in X$ denote by $d_{T}(x)$ the so-called modulus of decrease of the function $x$ on the interval $[T, \infty)$, defined by the formula

$$
d_{T}(x)=\sup \{|x(t)-x(s)|-[x(t)-x(s)]: T \leq s<t\} .
$$

Further, let us put

$$
\begin{gathered}
d_{T}(X)=\sup \left\{d_{T}(x): x \in X\right\}, \\
d_{\infty}(X)=\lim _{T \rightarrow \infty} d_{T}(X) .
\end{gathered}
$$

In a similar way we may define the modulus of increase of function $x$ and the set $X$ (cf. [1]).

Finally, let us define the set quantity $\mu_{d}$ in the following way

$$
\mu_{d}(X)=\omega_{0}^{\infty}(X)+d_{\infty}(X)+\limsup _{t \rightarrow \infty} \operatorname{diam} X(t) .
$$

It can be shown (see [7]) that $\mu_{d}$ is the measure of noncompactness in the algebra $B C\left(\mathbb{R}_{+}\right)$. The kernel ker $\mu_{d}$ of this measure consists of all sets $X \in \mathfrak{M}_{B C\left(\mathbb{R}_{+}\right)}$such 
that functions belonging to $X$ are locally equicontinuous on $\mathbb{R}_{+}$and the thickness of the bundle $X(t)$ formed by functions from $X$ tends to zero at infinity. Moreover, all functions from $X$ are ultimately nondecreasing on $\mathbb{R}_{+}$(cf. [1] for details).

Now we recall that the measure $\mu_{d}$ has following property.

Theorem 3.1. The measure of noncompactness $\mu_{d}$ defined by (3.1) satisfies condition $(m)$ on the family of all nonempty and bounded subsets $X$ of Banach algebra $B C\left(\mathbb{R}_{+}\right)$ such that functions belonging to $X$ are nonnegative on $\mathbb{R}_{+}$.

The proof of above theorem may be found in [4].

Further, let us assume that $\Omega$ is a nonempty subset of the Banach algebra $B C\left(\mathbb{R}_{+}\right)$ and $F: \Omega \rightarrow B C\left(\mathbb{R}_{+}\right)$is an operator. Consider the operator equation

$$
x(t)=(F x)(t), \quad t \in \mathbb{R}_{+},
$$

where $x \in \Omega$.

Definition 3.2. [1] We say that solutions of Eq. (3.2) are asymptotically stable if there exists a ball $B\left(x_{0}, r\right)$ in $B C\left(\mathbb{R}_{+}\right)$such that $B\left(x_{0}, r\right) \cap \Omega \neq \phi$ and for each $\varepsilon>0$ there exists $T>0$ such that $|x(t)-y(t)| \leq \varepsilon$ for all solutions $x, y \in B\left(x_{0}, r\right) \cap \Omega$ of Eq. (3.2) and for $t \geq T$.

Let us mention that the measure of noncompactness $\mu_{d}$ defined by formula (3.1) allows us to characterize solutions of considered operator equations in terms of the concept of asymptotic stability. Namely, if solutions of an operator equation considered in the algebra $B C\left(\mathbb{R}_{+}\right)$belong to a bounded subset being a member of the family ker $\mu_{d}$, then from the previously given description of the kernel ker $\mu_{d}$ we infer that those solutions are asymptotically stable in the sense of Definition 3.2 (cf. also Remark 2.5).

\section{The existence of asymtotically stable and ulti- mately nondecreasing solutions of a quadratic fractional integral equation in the Banach algebra $B C\left(\mathbb{R}_{+}\right)$}

In this section we will consider the solvability of some functional integral equation in the Banach algebra $B C\left(\mathbb{R}_{+}\right)$. Using the technique of measures of noncompactness we will prove that this equation has solutions on an unbounded interval. Moreover, we will also obtain some characterization of those solutions. Obviously, we will apply the measure of noncompactness described in the previous section.

In our considerations we will often use the so-called superposition operator. In order to define that operator we assume that $J \subset \mathbb{R}$ is an interval and consider a set $X_{J}$ consisting of all functions $x: \mathbb{R}_{+} \rightarrow J$. Moreover, $f: \mathbb{R}_{+} \times J \rightarrow \mathbb{R}$ is a given function. Then, for every function $x \in X_{J}$ we may assign the function $F x$ defined by the formula

$$
(F x)(t)=f(t, x(t))
$$


for $t \in \mathbb{R}_{+}$. The operator $F$ defined in such a way is called the superposition operator generated by the function $f$ (cf. [2]).

The below quoted lemma [1] presents a useful property of the superposition operator which is considered in the Banach space $B\left(\mathbb{R}_{+}\right)$consisting of all real functions defined and bounded on $\mathbb{R}_{+}$. Obviously, the space $B\left(\mathbb{R}_{+}\right)$is equipped with the classical supremum norm. Since $B\left(\mathbb{R}_{+}\right)$has the structure of a Banach algebra, we can consider the Banach algebra $B C\left(\mathbb{R}_{+}\right)$as a subalgebra of $B\left(\mathbb{R}_{+}\right)$.

Lemma 4.1. Assume that the following hypotheses are satisfied:

$(\alpha)$ The function $f$ is continuous on the set $\mathbb{R}_{+} \times J$.

( $\beta)$ The function $t \mapsto f(t, u)$ is ultimately nondecreasing uniformly with respect to $u$ belonging to bounded subintervals of $J$, i.e.,

$$
\lim _{T \rightarrow \infty}\left\{\sup \left\{|f(t, u)-f(s, u)|-[f(t, u)-f(s, u)]: t>s \geq T, u \in J_{1}\right\}\right\}=0
$$

for any bounded subinterval $J_{1} \subseteq J$.

$(\gamma)$ For any fixed $t \in \mathbb{R}_{+}$the function $u \mapsto f(t, u)$ is nondecreasing on $J$.

$(\delta)$ The function $u \mapsto f(t, u)$ satisfies a Lipschitz condition, i.e., there exist a constant $k>0$ such that

$$
|f(t, u)-f(t, v)| \leq k|u-v|
$$

for all $t \geq 0$ and all $u, v \in J$.

Then the inequality

$$
d_{\infty}(F x) \leq k d_{\infty}(x)
$$

holds for any function $x \in X_{J} \cap B\left(\mathbb{R}_{+}\right)$, where $k$ is the Lipschitz constant from assumption $(\delta)$.

Observe that in view of the remark mentioned previously the above lemma is also valid in the Banach algebra $B C\left(\mathbb{R}_{+}\right)$.

As we announced before, in this section we will investigate the existence of solutions of the quadratic fractional integral equation having the form

$$
x(t)=\left(U_{1} x\right)(t)\left(U_{2} x\right)(t),
$$

where

$$
\left(U_{i} x\right)(t)=m_{i}(t)+f_{i}(t, x(t)) \int_{0}^{t} \frac{v_{i}(t, s, x(s))}{(t-s)^{\alpha_{i}}} d s
$$

for $t \in \mathbb{R}_{+}$and $i=1,2$. Here we assume that $\alpha_{i} \in(0,1)$ is a fixed number for $i=1,2$. Our investigations will be conducted in the Banach algebra $B C\left(\mathbb{R}_{+}\right)$. For further purposes we define the few operators on the space $B C\left(\mathbb{R}_{+}\right)$by putting:

$$
\left(F_{i} x\right)(t)=f_{i}(t, x(t)),
$$




$$
\left(V_{i} x\right)(t)=\int_{0}^{t} \frac{v_{i}(t, s, x(s))}{(t-s)^{\alpha_{i}}} d s
$$

for $i=1,2$. Obviously, we have

$$
\left(U_{i} x\right)(t)=m_{i}(t)+\left(F_{i} x\right)(t)\left(V_{i} x\right)(t)
$$

for $i=1,2$ and for $t \in \mathbb{R}_{+}$. Moreover, let us introduce the following sets:

$$
\begin{gathered}
\Delta:=\left\{(t, s) \in \mathbb{R}_{+} \times \mathbb{R}_{+}: s \leq t\right\}, \\
A:=\left\{(t, s, x) \in \mathbb{R}_{+} \times \mathbb{R}_{+} \times \mathbb{R}: s \leq t\right\}, \\
A_{+}:=\left\{(t, s, x) \in \mathbb{R}_{+} \times \mathbb{R}_{+} \times \mathbb{R}_{+}: s \leq t\right\} .
\end{gathered}
$$

We will study Eq. (4.1) under following assumptions.

(i) The function $m_{i}$ is nonnegative, bounded, continuous and ultimately nondecreasing $(i=1,2)$.

(ii) The function $f_{i}: \mathbb{R}_{+} \times \mathbb{R}_{+} \rightarrow \mathbb{R}_{+}$satisfies the conditions $(\alpha)-(\gamma)$ of Lemma 4.1. Moreover, the function $t \mapsto f_{i}(t, 0)$ is bounded for $i=1,2$.

(iii) The function $f_{i}(i=1,2)$ satisfies the Lipschitz condition with respect to the second variable, i.e., there exists a constant $k_{i}>0$ such that

$$
\left|f_{i}(t, x)-f_{i}(t, y)\right| \leq k_{i}|x-y|
$$

for $x, y, t \in \mathbb{R}_{+}(i=1,2)$.

(iv) $v_{i}: A \rightarrow \mathbb{R}$ is a continuous function such that $v_{i}: A_{+} \rightarrow \mathbb{R}_{+}(i=1,2)$. Moreover, there exists a continuous and nondecreasing function $G_{i}: \mathbb{R}_{+} \rightarrow \mathbb{R}_{+}$ and a bounded and continuous function $g_{i}: \Delta \rightarrow \mathbb{R}_{+}$such that $v_{i}(t, s, x)=$ $g_{i}(t, s) G_{i}(|x|)$ for $t, s \in \mathbb{R}_{+}(s \leq t), x \in \mathbb{R}$ and $i=1,2$.

(v) The following property holds

$$
\lim _{t \rightarrow \infty} \int_{0}^{t} \frac{g_{i}(t, s)}{(t-s)^{\alpha_{i}}} d s=0
$$

for $i=1,2$.

In view of above assumptions we may define following constants $(i=1,2)$ :

$$
\begin{gathered}
\bar{F}_{i}=\sup \left\{\left|f_{i}(t, 0)\right|: t \in \mathbb{R}_{+}\right\}, \\
\bar{G}_{i}=\sup \left\{\int_{0}^{t} \frac{g_{i}(t, s)}{(t-s)^{\alpha_{i}}} d s: t \in \mathbb{R}_{+}\right\}, \\
\bar{g}_{i}=\sup \left\{g_{i}(t, s): t, s \in \mathbb{R}_{+}\right\}, \\
\bar{F}=\max \left\{\bar{F}_{1}, \bar{F}_{2}\right\}, \\
k=\max \left\{k_{1}, k_{2}\right\}, \\
m=\max \left\{\left\|m_{1}\right\|,\left\|m_{2}\right\|\right\} .
\end{gathered}
$$

The last assumption has the form: 
(vi) There exists a solution $r_{0}>0$ of the inequality

$$
\left[m+k \bar{G}_{1} r G_{1}(r)+\bar{F} \bar{G}_{1} G_{1}(r)\right]\left[m+k \bar{G}_{2} r G_{2}(r)+\bar{F} \bar{G}_{2} G_{2}(r)\right] \leq r
$$

such that

$$
\begin{aligned}
& m k\left(\bar{G}_{1} G_{1}\left(r_{0}\right)+\bar{G}_{2} G_{2}\left(r_{0}\right)\right)+2 k \bar{F} \bar{G}_{1} G_{1}\left(r_{0}\right) \bar{G}_{2} G_{2}\left(r_{0}\right) \\
& +2 k^{2} r_{0} \bar{G}_{1} G_{1}\left(r_{0}\right) \bar{G}_{2} G_{2}\left(r_{0}\right)<1 .
\end{aligned}
$$

The existence result concerning the functional integral equation (4.1) is contained in the below given theorem.

Theorem 4.2. Under assumptions (i)-(vi) Eq. (4.1) has at least one solution $x=x(t)$ in the space $B C\left(\mathbb{R}_{+}\right)$which is nonnegative, asymptotically stable and ultimately nondecreasing.

Proof. Let us assume that $\Omega$ is the subset of the Banach algebra $B C\left(\mathbb{R}_{+}\right)$consisting of all functions being nonnegative on $\mathbb{R}_{+}$. We will consider operators $V_{i}(i=1,2)$ on the set $\Omega$. Now, fix an arbitrary function $x \in \Omega$. Then, in virtue of assumptions (i), (ii) and (iv) we derive that the function $U_{i} x$ is nonnegative on $\mathbb{R}_{+}(i=1,2)$.

Next, in view of (4.2) and the imposed assumptions, we obtain:

$$
\begin{aligned}
\left(U_{i} x\right)(t) & \leq m_{i}(t)+\left[k_{i} x(t)+f_{i}(t, 0)\right] \int_{0}^{t} \frac{v_{i}(t, s, x(s))}{(t-s)^{\alpha_{i}}} d s \\
& \leq m_{i}(t)+\left[k_{i} x(t)+f_{i}(t, 0)\right] G_{i}(\|x\|) \int_{0}^{t} \frac{g_{i}(t, s)}{(t-s)^{\alpha_{i}}} d s \\
& \leq\left\|m_{i}\right\|+k_{i} \bar{G}_{i}\|x\| G_{i}(\|x\|)+\bar{F} \bar{G}_{i} G_{i}(\|x\|)
\end{aligned}
$$

for $t \in \mathbb{R}_{+}, i=1,2$.

This estimate yields that the function $U_{i} x$ is bounded on $\mathbb{R}_{+}(i=1,2)$.

Next, let us observe that in view of the properties of the superposition operator [2] and assumption (ii) we infer that the function $F_{i} x$ is continuous on $\mathbb{R}_{+}(i=1,2)$. Therefore, in order to show that $U_{i} x$ is continuous on the interval $\mathbb{R}_{+}$it is sufficient to show that the function $V_{i} x$ is continuous on $\mathbb{R}_{+}$.

To this end let us fix $T>0$ and $\varepsilon>0$ and choose arbitrarily $t, s \in[0, T]$ such that 
$|t-s| \leq \varepsilon$. Without loss of generality we may assume that $s<t$. Then we obtain

$$
\begin{aligned}
\left|\left(V_{i} x\right)(t)-\left(V_{i} x\right)(s)\right| & \leq\left|\int_{0}^{t} \frac{v_{i}(t, \tau, x(\tau))}{(t-\tau)^{\alpha_{i}}} d \tau-\int_{0}^{t} \frac{v_{i}(s, \tau, x(\tau))}{(t-\tau)^{\alpha_{i}}} d \tau\right| \\
& +\left|\int_{0}^{t} \frac{v_{i}(s, \tau, x(\tau))}{(t-\tau)^{\alpha_{i}}} d \tau-\int_{0}^{s} \frac{v_{i}(s, \tau, x(\tau))}{(t-\tau)^{\alpha_{i}}} d \tau\right| \\
& +\left|\int_{0}^{s} \frac{v_{i}(s, \tau, x(\tau))}{(t-\tau)^{\alpha_{i}}} d \tau-\int_{0}^{s} \frac{v_{i}(s, \tau, x(\tau))}{(s-\tau)^{\alpha_{i}}} d \tau\right| \\
& \leq \int_{0}^{t} \frac{\left|v_{i}(t, \tau, x(\tau))-v_{i}(s, \tau, x(\tau))\right|}{(t-\tau)^{\alpha_{i}}} d \tau \int_{s}^{t} \frac{v_{i}(s, \tau, x(\tau))}{(t-\tau)^{\alpha_{i}}} d \tau \\
& +\int_{0}^{s} v_{i}(s, \tau, x(\tau))\left[\frac{1}{(s-\tau)^{\alpha_{i}}}-\frac{1}{(t-\tau)^{\alpha_{i}}}\right] d \tau \\
& \left.\leq \omega_{\|x\|}^{T}\left(v_{i}, \varepsilon\right) \int_{0}^{t} \frac{1}{(t-\tau)^{\alpha_{i}}} d \tau+G_{i}(\|x\|)^{\prime}\right]_{s}^{t} \frac{1}{(t-\tau)^{\alpha_{i}}} d \tau \\
& +G_{i}(\|x\|) \bar{g}_{i} \int_{0}^{s}\left[\frac{1}{(s-\tau)^{\alpha_{i}}}-\frac{1}{(t-\tau)^{\alpha_{i}}}\right] d \tau \\
& \leq \omega_{\|x\|}^{T}\left(v_{i}, \varepsilon\right) \frac{t^{1-\alpha_{i}}}{1-\alpha_{i}}+G_{i}(\|x\|) \bar{g}_{i} \frac{(t-s)^{1-\alpha_{i}}}{1-\alpha_{i}} \\
& +G_{i}(\|x\|) \bar{g}_{i}\left[\frac{s^{1-\alpha_{i}}}{1-\alpha_{i}}-\frac{t^{1-\alpha_{i}}}{1-\alpha_{i}}+\frac{(t-s)^{1-\alpha_{i}}}{1-\alpha_{i}}\right] \\
& \leq \omega_{\|x\|}^{T}\left(v_{i}, \varepsilon\right) \frac{T^{1-\alpha_{i}}}{1-\alpha_{i}}+2 G_{i}(\|x\|) \bar{g}_{i} \frac{\varepsilon^{1-\alpha_{i}}}{1-\alpha_{i}},
\end{aligned}
$$

where we denoted

$\omega_{d}^{T}\left(v_{i}, \varepsilon\right)=\sup \left\{\left|v_{i}(t, \tau, x)-v_{i}(s, \tau, x)\right|: t, s, \tau \in[0, T],|t-s| \leq \varepsilon, x \in[-d, d]\right\}$.

From the above estimate and the fact that function $v_{i}$ is uniformly continuous on the set $\left\{(t, s, y) \in \mathbb{R}^{3}: 0 \leq s \leq t \leq T, y \in[-\|x\|,\|x\|]\right\}$ we have that the last part of above estimate tends to zero as $\varepsilon \rightarrow 0$ and this implies the continuity of the function $V_{i} x$. Gathering the above established facts and estimate (4.4) we conclude that the operator $U_{i}(i=1,2)$ transforms the set $\Omega$ into itself.

Apart from this, in view of (4.3) and assumption (vi) we infer that there exists a number $r_{0}>0$ such that the operator $S=U_{1} U_{2}$ transforms into itself the set $\Omega_{r_{0}}$ defined in the following way

$$
\Omega_{r_{0}}=\left\{x \in B C\left(\mathbb{R}_{+}\right): 0 \leq x(t) \leq r_{0} \text { for } t \in \mathbb{R}_{+}\right\} .
$$

Moreover, the following inequality is satisfied

$$
\left\|U_{i} \Omega_{r_{0}}\right\| \leq m+k_{i} \bar{G} r_{0} G_{i}\left(r_{0}\right)+\bar{F} \bar{G} G_{i}\left(r_{0}\right) .
$$

In the sequel we will work with the measure of noncompactness $\mu_{d}$. Thus, let us fix a nonempty subset $X$ of the set $\Omega_{r_{0}}$ and choose arbitrary numbers $T>0$ and 
$\varepsilon>0$. Then, for $x \in X$ and for $t, s \in[0, T]$ such that $|t-s| \leq \varepsilon$ and $t \geq s$ we have

$$
\begin{aligned}
\left|\left(U_{i} x\right)(t)-\left(U_{i} x\right)(s)\right| & \leq \omega^{T}\left(m_{i}, \varepsilon\right)+\left|\left(F_{i} x\right)(t)\left(V_{i} x\right)(t)-\left(F_{i} x\right)(s)\left(V_{i} x\right)(s)\right| \\
& \leq \omega^{T}\left(m_{i}, \varepsilon\right)+\left|\left(F_{i} x\right)(t)\left(V_{i} x\right)(t)-\left(F_{i} x\right)(s)\left(V_{i} x\right)(t)\right| \\
& +\left|\left(F_{i} x\right)(s)\left(V_{i} x\right)(t)-\left(F_{i} x\right)(s)\left(V_{i} x\right)(s)\right| \\
& \leq \omega^{T}\left(m_{i}, \varepsilon\right)+\left|\left(F_{i} x\right)(t)-\left(F_{i} x\right)(s)\right|\left|\left(V_{i} x\right)(t)\right| \\
& +\left|\left(F_{i} x\right)(s)\right|\left|\left(V_{i} x\right)(t)-\left(V_{i} x\right)(s)\right| .
\end{aligned}
$$

Reasoning similarly we derive the estimate

$$
\begin{aligned}
\left|\left(F_{i} x\right)(t)-\left(F_{i} x\right)(s)\right| & \leq\left|f_{i}(t, x(t))-f_{i}(t, x(s))\right|+\left|f_{i}(t, x(s))-f_{i}(s, x(s))\right| \\
& \leq k_{i}|x(t)-x(s)|+\left|f_{i}(t, x(s))-f_{i}(s, x(s))\right| \\
& \leq k_{i} \omega^{T}(x, \varepsilon)+\omega_{\|x\|}^{T}\left(f_{i}, \varepsilon\right)
\end{aligned}
$$

where we denoted

$\omega_{d}^{T}\left(f_{i}, \varepsilon\right)=\sup \left\{\left|f_{i}(t, x)-f_{i}(s, x)\right|: t, s \in[0, T],|t-s| \leq \varepsilon, x \in[-d, d]\right\}$.

Moreover, we have the following evaluations

$$
\begin{gathered}
\left|\left(V_{i} x\right)(t)\right| \leq G_{i}(\|x\|) \int_{0}^{t} \frac{g_{i}(t, \tau)}{(t-\tau)^{\alpha_{i}}} d \tau \leq G_{i}(\|x\|) \bar{G}_{i}, \\
\left|\left(F_{i} x\right)(s)\right| \leq k_{i}|x(s)|+\left|f_{i}(s, 0)\right| \leq k_{i} r_{0}+\bar{F}_{i},
\end{gathered}
$$

which hold for arbitrary $t, s \in \mathbb{R}_{+}$and $i=1,2$.

Further, linking (4.5), (4.6) with the above obtained evaluations, we infer that the following estimate holds

$$
\begin{aligned}
\left|\left(U_{i} x\right)(t)-\left(U_{i} x\right)(s)\right| & \leq \omega^{T}\left(m_{i}, \varepsilon\right)+\left[k_{i} \omega^{T}(x, \varepsilon)+\omega_{\|x\|}^{T}\left(f_{i}, \varepsilon\right)\right] G_{i}\left(\|x\| \bar{G}_{i}\right. \\
& +\left[k_{i} r_{0}+\bar{F}_{i}\right]\left[\omega_{\|x\|}^{T}\left(v_{i}, \varepsilon\right) \frac{T^{1-\alpha_{i}}}{1-\alpha_{i}}+2 G_{i}(\|x\|) \bar{g}_{i} \frac{\varepsilon^{1-\alpha_{i}}}{1-\alpha_{i}}\right] .
\end{aligned}
$$

Observe that the terms $\omega^{T}\left(m_{i}, \varepsilon\right), \omega_{\|x\|}^{T}\left(f_{i}, \varepsilon\right)$ and $\omega_{\|x\|}^{T}\left(v_{i}, \varepsilon\right)$ tend to zero as $\varepsilon \rightarrow 0$ since the functions $m_{i}, f_{i}$ and $v_{i}$ are uniformly continuous on the sets $[0, T],[0, T] \times$ $[-\|x\|,\|x\|]$ and $\left\{(t, s, y) \in \mathbb{R}^{3}: 0 \leq s \leq t \leq T, y \in[-\|x\|,\|x\|]\right\}$, respectively. Hence we obtain

$$
\omega_{0}^{T}\left(U_{i} X\right) \leq k_{i} \bar{G}_{i} G_{i}\left(r_{0}\right) \omega_{0}^{T}(X)
$$

and consequently

$$
\omega_{0}^{\infty}\left(U_{i} X\right) \leq k_{i} \bar{G}_{i} G_{i}\left(r_{0}\right) \omega_{0}^{\infty}(X) .
$$

In what follows, let us choose arbitrarily $x, y \in X$ and $t \in \mathbb{R}_{+}$. Then, applying 
our assumptions, we derive

$$
\begin{aligned}
& \left|\left(U_{i} x\right)(t)-\left(U_{i} y\right)(t)\right| \\
& \leq\left|f_{i}(t, x(t))-f_{i}(t, y(t))\right| \int_{0}^{t} \frac{v_{i}(t, \tau, x(\tau))}{(t-\tau)^{\alpha_{i}}} d \tau \\
& +f_{i}(t, y(t)) \int_{0}^{t} \frac{\left|v_{i}(t, \tau, x(\tau))-v_{i}(t, \tau, y(\tau))\right|}{(t-\tau)^{\alpha_{i}}} d \tau \\
& \leq k_{i}|x(t)-y(t)| G_{i}(\|x\|) \int_{0}^{t} \frac{g_{i}(t, \tau)}{(t-\tau)^{\alpha_{i}}} d \tau \\
& +\left[k_{i} y(t)+f_{i}(t, 0)\right] \int_{0}^{t} \frac{g_{i}(t, \tau)\left[G_{i}(x(\tau))-G_{i}(y(\tau))\right]}{(t-\tau)^{\alpha_{i}}} d \tau \\
& \leq k_{i}|x(t)-y(t)| G_{i}\left(r_{0}\right) \int_{0}^{t} \frac{g_{i}(t, \tau)}{(t-\tau)^{\alpha_{i}}} d \tau+\left[k_{i} r_{0}+\bar{F}_{i}\right] 2 G_{i}\left(r_{0}\right) \int_{0}^{t} \frac{g_{i}(t, \tau)}{(t-\tau)^{\alpha_{i}}} d \tau
\end{aligned}
$$

Hence, keeping in mind of assumption (v), we obtain the following equality

$$
\limsup _{t \rightarrow \infty} \operatorname{diam}\left(U_{i} X\right)(t)=0 .
$$

Now, we show that $U_{i}$ is continuous on the set $\Omega_{r_{0}}$. To this end fix $\varepsilon>0$ and take $x, y \in \Omega_{r_{0}}$ such that $\|x-y\| \leq \delta$. In view of (4.8) we know that we may find a number $T>0$ such that for arbitrary $t \geq T$ we get $\left|\left(U_{i} x\right)(t)-\left(U_{i} y\right)(t)\right| \leq \varepsilon$. On the other hand, if we take $t \in[0, T]$, we have

$$
\begin{aligned}
\left|\left(U_{i} x\right)(t)-\left(U_{i} y\right)(t)\right| & \leq\left|f_{i}(t, x(t))-f_{i}(t, y(t))\right| \int_{0}^{t} \frac{v_{i}(t, \tau, x(\tau))}{(t-\tau)^{\alpha_{i}}} d \tau \\
& +f_{i}(t, x(t)) \int_{0}^{t} \frac{\left|v_{i}(t, \tau, x(\tau))-v_{i}(t, \tau, y(\tau))\right|}{(t-\tau)^{\alpha_{i}}} d \tau \\
& \leq k_{i}|x(t)-y(t)| G_{i}(\|x\|) \int_{0}^{t} \frac{g_{i}(t, \tau)}{(t-\tau)^{\alpha_{i}}} d \tau \\
& +\left[k_{i}|y(t)|+f_{i}(t, 0)\right] \xi_{r_{0}}^{T}\left(v_{i}, \delta\right) \int_{0}^{t} \frac{d \tau}{(t-\tau)^{\alpha_{i}}} \\
& \leq k \delta \bar{G}_{i} G_{i}\left(r_{0}\right)+\left(k r_{0}+\bar{F}\right) \frac{T^{1-\alpha_{i}}}{1-\alpha_{i}} \xi_{r_{0}}^{T}\left(v_{i}, \delta\right)
\end{aligned}
$$

where we denoted

$\xi_{d}^{T}\left(v_{i}, \delta\right)=\sup \left\{\left|v_{i}(t, s, x)-v_{i}(t, s, y)\right|: t, s \in[0, T], x, y \in[-d, d],|x-y| \leq \delta\right\}$.

In view of the uniform continuity of the function $v_{i}$ on the set $\left\{(t, s, y) \in \mathbb{R}^{3}: 0 \leq\right.$ $\left.s \leq t \leq T, y \in\left[-r_{0}, r_{0}\right]\right\}$ we have that $\xi_{r_{0}}^{T}\left(v_{i}, \delta\right) \rightarrow 0$ as $\delta \rightarrow 0$. This yields that the last term in the above estimate is sufficiently small for $i=1,2$.

Next, fix arbitrarily $T>0$ and choose $t, s$ such that $t>s \geq T$. Then we have

$$
0 \leq\left|\frac{g_{i}(t, \tau)}{(t-\tau)^{\alpha_{i}}}-\frac{g_{i}(s, \tau)}{(s-\tau)^{\alpha_{i}}}\right|-\left[\frac{g_{i}(t, \tau)}{(t-\tau)^{\alpha_{i}}}-\frac{g_{i}(s, \tau)}{(s-\tau)^{\alpha_{i}}}\right] \leq 2 \frac{g_{i}(s, \tau)}{(s-\tau)^{\alpha_{i}}}
$$


Using (v) we obtain

$$
\lim _{T \rightarrow \infty}\left\{\sup \left\{\int_{0}^{s}\left\{\left|\frac{g_{i}(t, \tau)}{(t-\tau)^{\alpha_{i}}}-\frac{g_{i}(s, \tau)}{(s-\tau)^{\alpha_{i}}}\right|-\left[\frac{g_{i}(t, \tau)}{(t-\tau)^{\alpha_{i}}}-\frac{g_{i}(s, \tau)}{(s-\tau)^{\alpha_{i}}}\right]\right\} d \tau: T \leq s<t\right\}\right\}=0 .
$$

Then, for an arbitrary $x \in X$ we obtain

$$
\begin{aligned}
& \left|\left(U_{i} x\right)(t)-\left(U_{i} x\right)(s)\right|-\left[\left(U_{i} x\right)(t)-\left(U_{i} x\right)(s)\right] \\
& \leq\left|m_{i}(t)-m_{i}(s)\right|-\left[m_{i}(t)-m_{i}(s)\right]+\left|\left(F_{i} x\right)(t)\left(V_{i} x\right)(t)-\left(F_{i} x\right)(s)\left(V_{i} x\right)(t)\right| \\
& +\left|\left(F_{i} x\right)(s)\left(V_{i} x\right)(t)-\left(F_{i} x\right)(s)\left(V_{i} x\right)(s)\right|-\left[\left(F_{i} x\right)(t)\left(V_{i} x\right)(t)-\left(F_{i} x\right)(s)\left(V_{i} x\right)(t)\right] \\
& -\left[\left(F_{i} x\right)(s)\left(V_{i} x\right)(t)-\left(F_{i} x\right)(s)\left(V_{i} x\right)(s)\right] \\
& \leq d_{T}\left(m_{i}\right)+d_{T}\left(F_{i} x\right)\left(V_{i} x\right)(t) \\
& +\left(F_{i} x\right)(s)\left\{\left|\left(V_{i} x\right)(t)-\left(V_{i} x\right)(s)\right|-\left[\left(V_{i} x\right)(t)-\left(V_{i} x\right)(s)\right]\right\} .
\end{aligned}
$$

On the other hand we get

$$
\begin{aligned}
& \left|\left(V_{i} x\right)(t)-\left(V_{i} x\right)(s)\right|-\left[\left(V_{i} x\right)(t)-\left(V_{i} x\right)(s)\right] \\
& \leq\left|\int_{0}^{s} \frac{g_{i}(t, \tau) G_{i}(x(\tau))}{(t-\tau)^{\alpha_{i}}} d \tau-\int_{0}^{s} \frac{g_{i}(s, \tau) G_{i}(x(\tau))}{(s-\tau)^{\alpha_{i}}} d \tau\right|+\left|\int_{s}^{t} \frac{g_{i}(t, \tau) G_{i}(x(\tau))}{(t-\tau)^{\alpha_{i}}} d \tau\right| \\
& -\left[\int_{0}^{s} \frac{g_{i}(t, \tau) G_{i}(x(\tau))}{(t-\tau)^{\alpha_{i}}} d \tau-\int_{0}^{s} \frac{g_{i}(s, \tau) G_{i}(x(\tau))}{(s-\tau)^{\alpha_{i}}} d \tau\right]-\int_{s}^{t} \frac{g_{i}(t, \tau) G_{i}(x(\tau))}{(t-\tau)^{\alpha_{i}}} d \tau \\
& \leq \int_{0}^{s}\left|\frac{g_{i}(t, \tau) G_{i}(x(\tau))}{(t-\tau)^{\alpha_{i}}} d \tau-\frac{g_{i}(s, \tau) G_{i}(x(\tau))}{(s-\tau)^{\alpha_{i}}}\right| d \tau \\
& -\int_{0}^{s}\left[\frac{g_{i}(t, \tau) G_{i}(x(\tau))}{(t-\tau)^{\alpha_{i}}} d \tau-\frac{g_{i}(s, \tau) G_{i}(x(\tau))}{(s-\tau)^{\alpha_{i}}}\right] d \tau \\
& \leq G_{i}(\|x\|) \int_{0}^{s}\left\{\left|\frac{g_{i}(t, \tau)}{(t-\tau)^{\alpha_{i}}}-\frac{g_{i}(s, \tau)}{(s-\tau)^{\alpha_{i}}}\right|-\left[\frac{g_{i}(t, \tau)}{(t-\tau)^{\alpha_{i}}}-\frac{g_{i}(s, \tau)}{(s-\tau)^{\alpha_{i}}}\right]\right\} d \tau .
\end{aligned}
$$

Now, taking into account assumptions (i), (iv) and estimates (4.9), (4.10), we derive

$$
d_{\infty}\left(U_{i} x\right) \leq d_{\infty}\left(F_{i} x\right) G_{i}\left(r_{0}\right) \bar{G}_{i}
$$

for $i=1,2$. Hence, in view of Lemma 4.1, we derive the following inequality

$$
d_{\infty}\left(U_{i} x\right) \leq k_{i} \bar{G}_{i} G_{i}\left(r_{0}\right) d_{\infty}(x)
$$

for $i=1,2$. Further, combining the above inequality an (4.6), (4.7), (4.10), we obtain

$$
\mu_{d}\left(U_{i} X\right) \leq k_{i} \bar{G}_{i} G_{i}\left(r_{0}\right) \mu_{d}(X)
$$

for $i=1,2$.

Therefore, applying Theorem 2.4 we derive that the operator $S=U_{1} U_{2}$ is a contraction with respect to the measure of noncompactness $\mu_{d}$ with the constant $L$ given by the formula

$L=m k\left(\bar{G}_{1} G_{1}\left(r_{0}\right)+\bar{G}_{2} G_{2}\left(r_{0}\right)\right)+2 k \bar{F} \bar{G}_{1} G_{1}\left(r_{0}\right) \bar{G}_{2} G_{2}\left(r_{0}\right)+2 k^{2} r_{0} \bar{G}_{1} G_{1}\left(r_{0}\right) \bar{G}_{2} G_{2}\left(r_{0}\right)$. 
Observe that assumption (vi) implies that $L<1$. Thus, taking into account Theorem 2.4 we infer that the operator $S$ has at least one fixed point $x=x(t)$ belonging to the set $\Omega_{r_{0}}$. Moreover, in view of Remark 2.5 we conclude that $x$ is nonnegative on $\mathbb{R}_{+}$, asymptotically stable and ultimately nondecreasing.

This completes the proof.

Now we provide an example illustrating Theorem 4.2.

Example 4.3. Consider the quadratic fractional integral equation having form (4.1) with the operators $U_{1}, U_{2}$ defined by the following formulas

$$
\begin{gathered}
\left(U_{1} x\right)(t)=\frac{t}{3 t+1}+\arctan \left(t^{2}+x(t)\right) \int_{0}^{t} \frac{e^{-(t+s)} \sqrt{|x(t)|}}{(t-s)^{1 / 3}} d s, \\
\left(U_{2} x\right)(t)=\frac{1-e^{-t}}{4}+\frac{1}{2} \ln (x(t)+1) \int_{0}^{t} \frac{8 x^{4}(t)}{5(t+s+2)^{3}(t-s)^{1 / 5}} d s
\end{gathered}
$$

for $t \in \mathbb{R}_{+}$.

Observe that in this case the functions involved in Eq. (4.1) have the form:

$$
\begin{gathered}
m_{1}(t)=\frac{t}{3 t+1}, \quad m_{2}(t)=\frac{1-e^{-t}}{4}, \\
f_{1}(t, x)=\arctan \left(t^{2}+x\right), \quad f_{2}(t, x)=\frac{1}{2} \ln (x+1), \\
v_{1}(t, s, x)=e^{-(t+s)} \sqrt{|x|}, \\
v_{2}(t, s, x)=\frac{8 x^{4}}{5(t+s+2)^{3}} .
\end{gathered}
$$

Moreover, $\alpha_{1}=\frac{1}{3}, \alpha_{2}=\frac{1}{5}$.

It is easy to check that for the above functions there are satisfied assumptions of Theorem 4.2. Indeed, we have that the function $m_{i}=m_{i}(t)$ is nonnegative, bounded and continuous on $\mathbb{R}_{+}(i=1,2)$. Since $m_{1}$ and $m_{2}$ are increasing on $\mathbb{R}_{+}$we derive that they are also ultimately nondecreasing on $\mathbb{R}_{+}$. Moreover, $\left\|m_{1}\right\|=\frac{1}{3}$ and $\left\|m_{2}\right\|=\frac{1}{4}$. Thus, there is satisfied assumption (i). Further notice that the functions $f_{i}(i=1,2)$ transform continuously the set $\mathbb{R}_{+} \times \mathbb{R}_{+}$into $\mathbb{R}_{+}$. Moreover, $f_{1}$ is nondecreasing with respect to both variables and satisfies the Lipschitz condition (with respect to the second variable) with the constant $k_{1}=1$. Similarly, the function $f_{2}=f_{2}(t, x)$ is increasing with respect to $x$ and satisfies the Lipschitz condition with the constant $k_{2}=\frac{1}{2}$. Apart from this it is easily seen that $\bar{F}_{1}=\frac{\pi}{2}, \bar{F}_{2}=0$. Summing up, we see that functions $f_{1}$ and $f_{2}$ satisfy assumptions (ii) and (iii).

Next, let us note that the function $v_{i}$ is continuous on the set $A$ and transforms the set $A_{+}$into $\mathbb{R}_{+}$for $i=1,2$. Apart from this the function $v_{i}$ can be represented in the form $v_{i}(t, s, x)=g_{i}(t, s) G_{i}(|x|)(i=1,2)$, where $g_{1}(t, s)=e^{-(t+s)}, G_{1}(x)=\sqrt{|x|}$, $g_{2}(t, s)=\frac{8}{5(t+s+2)^{3}}, G_{2}(x)=x^{4}$. It is easily seen that assumption (iv) is satisfied for the functions $v_{1}$ and $v_{2}$. 
Further on, we have

$$
\int_{0}^{t} \frac{g_{1}(t, s)}{(t-s)^{\alpha_{1}}} d s=\int_{0}^{t} \frac{e^{-t-s}}{(t-s)^{1 / 3}} d s \leq e^{-t} \int_{0}^{t} \frac{d s}{(t-s)^{1 / 3}}=\frac{3}{2} e^{-t} t^{2 / 3} .
$$

Hence we see that

$$
\lim _{t \rightarrow \infty} \int_{0}^{t} \frac{g_{1}(t, s)}{(t-s)^{\alpha_{1}}} d s=0
$$

Moreover, we get

$$
\int_{0}^{t} \frac{g_{2}(t, s)}{(t-s)^{\alpha_{2}}} d s=\int_{0}^{t} \frac{8}{5(t+s+2)^{3}(t-s)^{1 / 5}} d s \leq \frac{8}{5(t+2)^{3}} \int_{0}^{t} \frac{d s}{(t-s)^{1 / 5}}=\frac{2 t^{4 / 5}}{(t+2)^{3}} .
$$

Thus, we have

$$
\lim _{t \rightarrow \infty} \int_{0}^{t} \frac{g_{2}(t, s)}{(t-s)^{\alpha_{2}}} d s=0 .
$$

This shows that assumption (v) is satisfied.

Finally, let us notice that taking into account the above estabilished facts we have that $m=\max \left\{\left\|m_{1}\right\|,\left\|m_{2}\right\|\right\}=\frac{1}{3}, k=\max \left\{k_{1}, k_{2}\right\}=1$ and $\bar{F}=\max \left\{\bar{F}_{1}, \bar{F}_{2}\right\}=\frac{\pi}{2}$. Thus, the first inequality from assumption (vi) has the form

$$
\left[\frac{1}{3}+\bar{G}_{1}\left(r \sqrt{r}+\frac{\pi}{2} r\right)\right]\left[\frac{1}{3}+\bar{G}_{2}\left(r^{5}+\frac{\pi}{2} r^{4}\right)\right] \leq r .
$$

It can be shown that the number $r_{0}=\frac{1}{2}$ is a solution of the above inequality such that it satisfies also the second inequality from assumption (vi).

Applying Theorem 4.2 we infer that the quadratic fractional integral equation considered in this example has a solution belonging to the set

$$
\Omega_{\frac{1}{2}}=\left\{x \in B C\left(\mathbb{R}_{+}\right): 0 \leq x(t) \leq \frac{1}{2} \text { for } t \in \mathbb{R}_{+}\right\},
$$

which is asymtotically stable and ultimately nondecreasing.

\section{References}

[1] J. Appell, J. Banaś and N. Merentes, Measures of noncompactnes in the study of asymptotically stable and ultimately nondecreasing solutions of integral equations, Zeit. Anal. Anwend. 29 (2010) 251-273.

[2] J. Appell, P.P. Zabrejko, Nonlinear Superposition Operators, Cambridge Univ. Press, Cambridge 1990.

[3] I.K. Argyros, Quadratic equations and applications to Chandrasekhar's and related equations, Bull. Austral. Math. Soc. 32 (1985) 275-282. 
[4] J. Banaś, S. Dudek, The technique of measures of noncompactness in Banach algebras and its applications to integral equations. Abstr. Appl. Anal., Volume 2013 (2013), Article ID 537897, 15 pages.

[5] J. Banaś, K. Goebel, Measures of Noncompactness in Banach Spaces, Lect. Notes Pure and Appl. Math. 60, Marcel Dekker, New York 1980.

[6] J. Banaś, M. Lecko, Fixed points of the product of operators in Banach algebra, Panamer. Math. J. 12 (2002) 101-109.

[7] J. Banaś, L. Olszowy, On a class of measures of noncompactnes in Banach algebras and their application to nonlinear integral equations, Zeit. Anal. Anwend. 28 (2009) 475-498.

[8] J. Banaś, D. O'Regan, On existence and local attractivity of solutions of a quadratic Volterra integral equation of fractional order, J. Math. Anal. Appl. 345 (2008) 573-582.

[9] J. Banaś, B. Rzepka, On existence and asymptotic stability of solutions of a nonlinear integral equation, J. Math. Anal. Appl. 284 (2003) 165-173.

[10] J. Banaś, B. Rzepka, Monotonic solutions of a quadratic integral equation of fractional order, J. Math. Anal. Appl. 322 (2007) 1371-1379.

[11] J. Banaś, K. Sadarangani, Solutions of some functional-integral equations in Banach algebra, Math. Comput. Modelling 38 (2003) 245-250.

[12] A. Ben Amar, S. Chouayekh and A. Jeribi, New fixed point theorems in Banach algebras under weak topology features and applications to nonlinear integral equations, J. Func. Anal. 259 (2010) 2215-2237.

[13] B. Cahlon, M. Eskin, Existence theorems for an integral equation of the Chandrasekhar H-equation with perturbation, J. Math. Anal. Appl. 83 (1981) 159-171.

[14] S. Chandrasekhar, Radiative Transfer, Oxford Univ. Press, London 1950.

[15] C. Corduneanu, Integral Equations and Applications, Cambridge Univ. Press, Cambridge 1991.

[16] M. Darwish, On quadratic integral equation of fractional orders, J. Math. Anal. Appl. 311 (2005) 112-119.

[17] K. Deimling, Nonlinear Functional Analysis, Springer Verlag, Berlin 1985.

[18] B.C. Dhage, On $\alpha$-condensing mappings in Banach algebras, The Math. Student 63 (1994) 146-152.

[19] B.C. Dhage, On a fixed point theorem in Banach algebras with applications, Appl. Math. Letters 18 (2005) 273-280.

[20] G. Gripenberg, On some epidemic models, Q. Appl. Math. 39 (1981) 317-327. 
[21] S. Hu, M. Kharani and W. Zhuang, Integral equations arising in the kinetic theory of gases, Appl. Analysis 34 (1989) 261-266.

\section{DOI: $10.7862 / \mathrm{rf} .2017 .5$}

\section{Szymon Dudek}

email: sdudek@prz.edu.pl

Department of Nonlinear Analysis

Rzeszów University of Technology

al. Powstańców Warszawy 8

35-959 Rzeszów

POLAND

Received 12.12.2016

Accepted 25.02.2017 